\title{
Influencia de la zona urbana de Macas en el Índice Calidad de Agua del río Jurumbaino.
}

\section{Influence Of The Urban Area Of Macas In The Water Quality Index Of The Jurumbaino River}

\author{
Rogelio Ureta Valdez . ${ }^{1}$, Patricio Méndez Zambrano. ${ }^{2}$ \&. Eduardo Cazar Rivera ${ }^{3}$
}

\section{Abstract. \\ DOI: https://doi.org/10.33262/cienciadigital.v3i3.1.679}

The water basins located in the vicinity of urban areas have been used for centuries for the benefit of the human being, which causes the waters of the rivers to be deteriorated, for this reason we have considered necessary the study of the river Jurumbaino, it is located next to the urban area of Morona and presents a deterioration in its waters, for this reason the main objective of this study is the determination of its quality implemented the water quality index ICA NSF which values both physical and chemical parameters, which were carried out both in situ and ex situ, the latter was carried out in the water quality laboratories of the Polytechnic School of Chimborazo, the sampling period was from March to June.

The three evaluated points according to the ICA NSF they present a general assessment of REGULAR, which is due to the high level of fecal coliforms found in their water due to two different The first is the contact with the urban area, and the second is the proximity to crops and grazing areas.

Keywords: Water quality, Anthropic, Jurumbaino, Macas.

\section{Resumen.}

Las cuencas hídricas ubicadas en la cercanía de las zonas urbanas han sido por siglos utilizadas para el beneficio del ser humano el mismo que causa que las aguas de los

1 Escuela Superior Politécnica de Chimborazo, Sede Morona Santiago, Ecuador. rogelio.ureta@espoch.edu.ec.

2 Escuela Superior Politécnica de Chimborazo, Sede Morona Santiago, Ecuador. patricio.mendez@espoch.edu.ec.

3 Escuela Superior Politécnica de Chimborazo, Sede Morona Santiago, Ecuador. eduardo.cazar@espoch.edu.ec 
ríos se vean deterioradas, por tal motivo hemos considerado necesario el estudio del rio Jurumbaino que está ubicado junto a la zona urbana del cantón Morona y presenta un deterioro en sus aguas, por tal motivo el objetivo principal de este estudio es la determinación de su calidad implementado el índice de calidad de agua ICA NSF el cual valora parámetros tanto físicos y químicos, los cuales se los realizó tanto in situ como ex situ, éste último se lo realizó en los laboratorios de calidad de agua de la Escuela Superior Politécnica de Chimborazo, el periodo de muestreo fue desde el mes de Marzo hasta Junio,

Los tres puntos evaluados según el ICA NSF presentan una valoración general de REGULAR el mismo que se debe al alto nivel de coliformes fecales encontrados en sus agua debido a dos variables la primero es el contacto con la zona Urbana, y el segundo es la cercanía con cultivos y zonas de pastoreo.

Palabras claves: Calidad del agua, Antrópico, Jurumbaino, Macas.

\section{Introducción.}

El agua es un compuesto indispensable para la vida de todas las especies vivientes no solo para el ser humano, y sin el agua la vida en la tierra no existiría o fuera muy escasa, el ser humano al formar una población o comunidad siempre busca aquellos lugares donde pueda tener un acceso libre a este recurso para establecer su población (Ramírez, 2011), por lo que el deterioro de las aguas es una problemática de orden social, ambiental y económico que dan como resultado serios impedimentos para llegar a tener un "Buen vivir". En el Ecuador se estima que hasta los 2000 m.s.n.m todas las fuentes de aguas están siendo deterioradas por intervenciones múltiples. (Isch, 2011)

La contaminación de las aguas superficiales se debe a dos aspectos de gran importancia el de origen natural (arrastre de material disuelto y particulado y existencia de materia orgánica natural) y el de origen antrópico (descargas de aguas residuales domésticas, escorrentía agrícola, efluentes de procesos industriales, etc.). (Torres et al., 2009).

A lo largo del tiempo los ríos han sido el blanco de un acelerado deterioro en su nivel de calidad esto es producto del mal uso de los mismos al ser utilizados como captadores de aguas residuales generadas en la zona urbana, industrial así como de la actividad agropecuaria (Fernández, 2017).

Se estima que el $90 \%$ de las aguas residuales de las ciudades y países en vía de desarrollo se descarga sin previo tratamiento en ríos, lagos y cursos de aguas costeras (Ibarra, 2007). Sin embargo una de las características de las aguas lóticas como las de un rio es la de la autodepuración; dependiendo del grado o intensidad de contaminación que presente el curso de agua, el flujo mismo del agua tiende a restituir de forma natural las condiciones que presentaba antes de la presencia del agente contaminante (Fernández, 2017). 
De acuerdo con lo ya mencionado el ingeniero debe estar preparado para tratar y solucionar, e implementar planes de control que eviten la acelerada contaminación del recurso agua, por lo que deberá plantear objetivos en beneficio de este tan importante recurso (Ramírez, 2011).Por tal motivo es indispensable el estudio de los ríos y causes que se ven afectados como el rio Jurumbaino que es el objeto de este estudio debido a la presencia de la zona urbana junto al rio, esta se puede ver afecta directa o indirectamente en la calidad del agua, ya que en las zonas cercanas al rio ha habido ausencia de sistema de alcantarillado por ende se evidencia la presencia de desechos y residuos sanitarios de una gran parte del barrio $27 \mathrm{de}$ febrero sobre el rio.

El índice de calidad de agua (ICA) es un método que nos ayuda en la determinación del nivel de calidad del agua independientemente de su origen; estas aguas pueden ser subterráneas o superficiales en un tiempo y espacio determinado. El ICA incluye diferentes parámetros tanto físicos, químicos como biológicos dentro de una ecuación matemática la cual nos arroja un resultado único el mismo con el que evaluaremos el estado de un cuerpo de agua.(Caho \& López 2017).

El objetivo de esta investigación es determinar el índice de calidad de agua del río Jurumbaino que se encuentra ubicado al oeste de la zona urbana del cantón Morona por medio del índice propuesto por la National Sanitation Foundation NSF(Torres et al., 2009).

El NSF es una organización fundada en 1944 con el fin de ayudar a estandarizar el saneamiento y la seguridad alimentaria (NSF Internacional, 2004) para lo cual se creó estándares independientes y programas de prueba que ayuden a mejorar la salud pública en general, el NSF analiza y certifica los productos para industrias del agua, alimentos, salud, así como también productos para el consumo humano, con el fin de disminuir los efectos negativos en la salud y proteger el medio ambiente. (Noticias Multinivel, 2019)

\section{Metodología.}

\section{Área de estudio y obtención de muestras.}

Este estudio fue realizado en el cantón Morona el cual según el último censo realizado por el INEC (instituto nacional de estadística y censo) cuenta con una población total de 41155 habitantes (INEC, 2010), el mismo que tiene una extensión de 46069 Km2 (GAD, 2016). El cantón presenta una temperatura promedio anual de $21,8^{\circ} \mathrm{C}$ y con una precipitación anual de 2612,0 mm (Instituto Nacional de Meteorología e Hidrología (INAMHI), 2016).

El área de estudio fue el rio Jurumbaino es uno de los ríos más representativos de la ciudad de Macas el cual fluye paralelamente a la ciudad, la toma de las muestras se las realizaron en los meses de marzo a junio del año 2019, para la estimación de los índices se obtuvo la información de tres puntos distintos los cuales están distribuidos a lo largo del área de estudio que comprenden un tramo de $6.93 \mathrm{~km}$. y de esta manera llegar a determinar las variables 
microbiológicas y fisicoquímicas que nos ayudaran a determinar el estado y calidad del agua, (González et al., 2013) para los puntos de muestreo se consideró la topografía del terreno es decir los puntos escogidos fueron de fácil acceso y considerando la posición con respecto a la intervención de la zona urbana a lo largo del área de estudio.

Tabla 1. Coordenadas UTM de los puntos de muestreo

\begin{tabular}{llccl}
\hline Punto & \multicolumn{1}{c}{ Descripción } & \multicolumn{1}{c}{ X } & \multicolumn{1}{c}{ Y } & \multicolumn{1}{c}{ Altura } \\
\hline 1 & Barrio La Florida & 819129 & 9746781 & 1050 m.s.n.m \\
2 & Puente Salida Al Quílamo & 819798 & 9745458 & 1005 m.s.n.m \\
3 & Puente Salida De Macas & 818166 & 9742579 & 934 m.s.n.m \\
\hline
\end{tabular}

Realizado por: Grupo de investigación.

\section{Descripción de los puntos de muestreo}

P1 (barrio la florida): inicio del tramo de estudio, como podemos observar en la fig.1 este punto fue considerado debido a que el caudal del rio aún no se ve intervenido por la zona urbana de Macas.

P2 (Puente Salida Al Quílamo): Punto intermedio en el que apreciamos el sitio con mayor contacto frente a la zona urbana por lo cual en nuestro estudio suponemos que es el lugar donde tendría ciertas desventajas en lo concerniente a la calidad del agua.

P3 (Puente Salida De Macas): Punto final del tramo de estudio, en el cual se localiza a salida de la zona urbana este se considera en la teoría

Ilustración 1. Área de estudio del Río Jurumbaino.

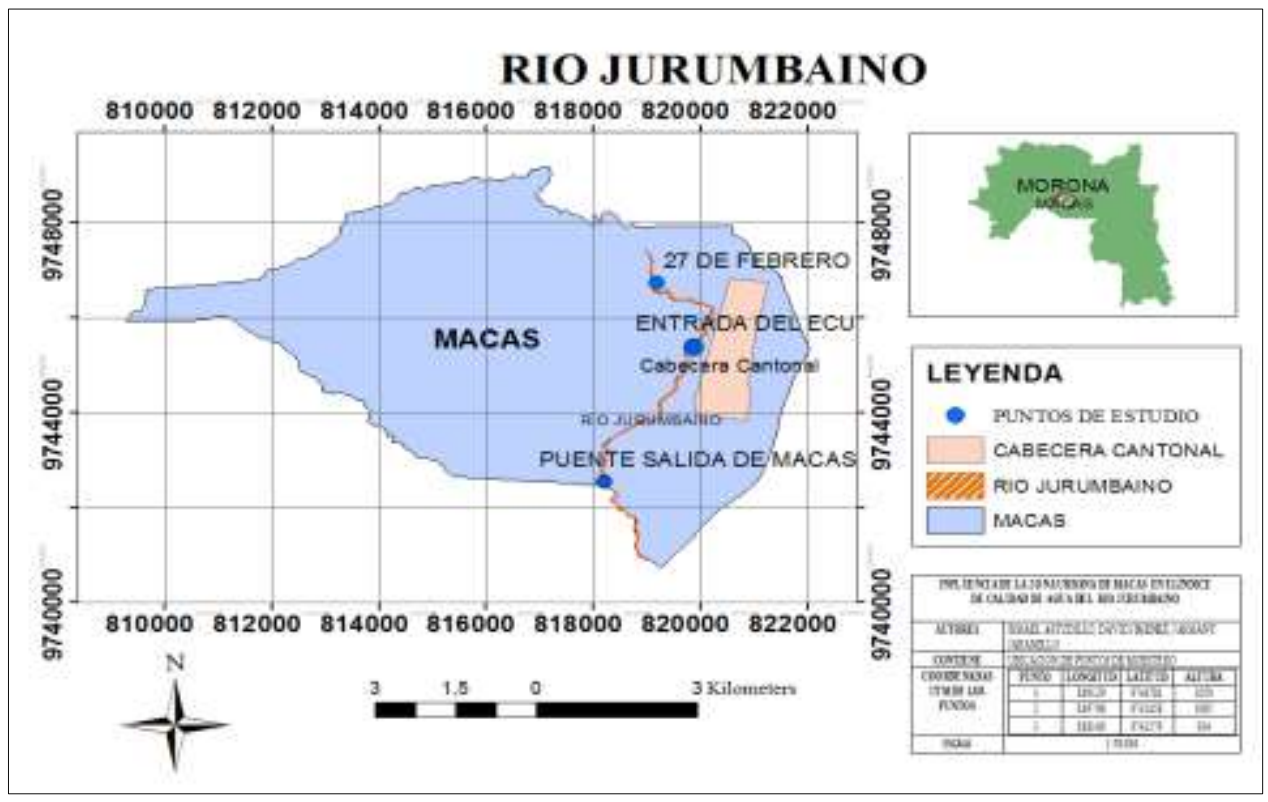

Fuente: Elaborado en ArcMap10.1. 
Para las mediciones de las variables in situ se determinó el $\mathrm{pH}$, Temperatura y oxígeno disuelto con la ayuda del equipo multiparamétrico modelo HQ40D portable, posteriormente se recolecto muestras de $1000 \mathrm{ml}$ por punto para los análisis fisicoquímicos (DBO5, solidos totales, nitratos (NO3), fosfatos (PO4), turbidez) y para los análisis microbiológicos se recolectaron muestras de $100 \mathrm{ml}$, los mismos que fueron determinados en el laboratorio de calidad de agua de la Escuela Superior Politécnica del Chimborazo. Todos estos parámetros son determinados con la ayuda del software IQA Data el mismo que aplica los índices establecidos por el NSF del ICA (Posselt \& Costa, 2015)

\section{Índice De Calidad De Agua}

La calidad de agua es determinada a partir de parámetros físicos químicos y biológicos en relación con su estado natural, la intervención humana y sus diversos usos. Por lo que utilizar un índice de calidad de agua nos facilita la interpretación de los datos ya que éstos índices reducen la cantidad de parámetros a utilizar, valorando así en una expresión simple (Fernández, 2009).

Cabe recalcar el hecho de que el NFC fue desarrollado en los Estados Unidos este puede aplicarse y adaptarse a múltiples estudios. (Castro et al., 2014)

\section{Determinación De Parámetros In situ}

La metodología utilizada en la etapa de campo fue mediante la utilización del multiparamétrico modelo HQ40D portable el mismo que ayuda a determinar cuatro parámetros que son medidos por tres sondas especializadas y calibradas las mismas que son introducidas en el punto de muestreo y utilizando como referencia los standard methods Ed 23, 2017 de los cuales el $\mathrm{pH}$ standard methods $4500-\mathrm{H}+\mathrm{A}$, Oxígeno disuelto standard methods 4500- OG, y por ultimo parámetro medido por el multiparamétrico que fue la temperatura standard methods 2550-B (Federation, 2017).

\section{Determinación De Parámetros Ex Situ (Laboratorio)}

Para la medición de algunos de los parámetros del índice de calidad de agua NSF fue necesario la recolección de muestras para el posterior análisis en el laboratorio de la Escuela Superior Politécnica de Chimborazo el mismo que cuenta con un laboratorio apto para la determinación de la calidad de agua en el cual se determinó según el standard methods Ed 23, 2017 los siguientes parámetros: Demanda bioquímica de oxígeno, Standard methods, 5210 B; Solidos totales, Standard methods 2540-B; Fosfatos Standard methods 4500-P-D; Turbidez Standard methods 2130-B; Nitratos Standard methods 4500-NO3-E; Coliformes fecales Standard methods 9221-B-E y F (Federation, 2017) 


\section{Resultados.}

Tabla 2. Resultados obtenidos mediante el programa IQAdata

\begin{tabular}{|c|c|c|c|c|c|c|}
\hline & $\begin{array}{l}\text { Barrio La } \\
\text { Florida }\end{array}$ & $\begin{array}{l}\text { Clasificación } \\
\text { ICA - NSF }\end{array}$ & $\begin{array}{l}\text { Puente } \\
\text { Salida Al } \\
\text { Quílamo }\end{array}$ & $\begin{array}{l}\text { Clasificación } \\
\text { ICA - NSF }\end{array}$ & $\begin{array}{l}\text { Puente } \\
\text { Salida De } \\
\text { Macas }\end{array}$ & $\begin{array}{l}\text { Clasificación } \\
\text { ICA - NSF }\end{array}$ \\
\hline $\begin{array}{l}\text { ler } \\
\text { muestreo }\end{array}$ & 59,86 & Regular & 61,46 & Regular & 61,32 & Regular \\
\hline $\begin{array}{l}2 \text { do } \\
\text { muestreo }\end{array}$ & 65,64 & Regular & 62,64 & Regular & 65,29 & Regular \\
\hline $\begin{array}{l}3 \text { er } \\
\text { muestreo }\end{array}$ & 58,19 & Regular & 56,43 & Regular & 51,22 & Regular \\
\hline
\end{tabular}

Realizado por: Grupo de investigación.

\section{Parámetros Fisicoquímicos Obtenidos}

A continuación, las tablas 4, 5 y 6, son los análisis físico-químicos, fueron obtenidos de los diferentes puntos de muestreo por 3 meses que duro el estudio, cabe destacar que el clima en el cual recolectamos las muestras era lluvioso, por lo cual creemos que afectara en los resultados analizados de alguna manera.

Tabla 3. Resultados Punto 1 Parámetros físico-químicos.

\begin{tabular}{llllll}
\hline \multicolumn{5}{c}{ Barrio La Florida } \\
\hline Parámetros & Unidad & Muestra 1 & Muestra 2 & Muestra 3 \\
\hline 1. & Oxí́geno disuelto & $\mathrm{mg} / \mathrm{l}$ & 7,36 & 8,78 & 7,1 \\
2. & $\mathrm{pH}$ & & 7,53 & 8,11 & 7,75 \\
3. & DBO & $\mathrm{mg} / \mathrm{l}$ & 4,2 & 4,3 & 8,2 \\
4. & Nitratos & $\mathrm{mg} / \mathrm{l}$ & 3,7 & 2,6 & 3,7 \\
5. & Coliformes fecales & $\mathrm{NMP} / 100 \mathrm{ml}$ & 2000 & 300 & 300 \\
6. & Temperatura & ${ }^{\circ} \mathrm{C}$ & 18,4 & 19,4 & 19,5 \\
7. & Turbidez & $\mathrm{NTU}$ & 16,8 & 13,1 & 27,8 \\
8. & Solidos totales & $\mathrm{gr}$ & 154 & 124 & 184 \\
9. & Fosfatos & $\mathrm{mg} / \mathrm{l}$ & 0,15 & 0,2 & 0,31 \\
\hline
\end{tabular}

Realizado por: Grupo de investigación. 
Tabla 4. Resultados Punto 2 Parámetros físico-químicos.

\begin{tabular}{llllll}
\hline \multicolumn{5}{c}{ Puente Salida Al Quílamo } \\
\hline Parámetros & Unidad & Muestra 1 & Muestra 2 & Muestra 3 \\
\hline 1. & Oxígeno disuelto & $\mathrm{mg} / \mathrm{l}$ & 6,96 & 7,57 & 6,7 \\
2. & $\mathrm{pH}$ & & 7,57 & 7,88 & 7,74 \\
3. & $\mathrm{DBO}$ & $\mathrm{mg} / \mathrm{l}$ & 1,6 & 3,93 & 7,1 \\
4. & Nitratos & $\mathrm{mg} / \mathrm{l}$ & 4,8 & 5,3 & 4 \\
5. & Coliformes fecales & $\mathrm{NMP} / 100 \mathrm{ml}$ & 1000 & 400 & 400 \\
6. & Temperatura & ${ }^{\circ} \mathrm{C}$ & 21,3 & 18,27 & 19,5 \\
7. & Turbidez & $\mathrm{NTU}$ & 22,8 & 31,6 & 31,7 \\
8. & Solidos totales & $\mathrm{gr}$ & 206 & 164 & 248 \\
9. & Fosfatos & $\mathrm{mg} / 1$ & 0,18 & 0,22 & 0,33 \\
\hline
\end{tabular}

Realizado por: Grupo de investigación.

Tabla 5. Resultados Punto 3 Parámetros físico-químicos

\begin{tabular}{llllll}
\hline \multicolumn{5}{c}{ Puente Salida De Macas } \\
\hline Parámetros & Unidad & Muestra 1 & Muestra 2 & Muestra 3 \\
\hline 1. & Oxígeno disuelto & $\mathrm{mg} / 1$ & 6,89 & 8,11 & 7,5 \\
2. & $\mathrm{pH}$ & & 7,62 & 8 & 7,71 \\
3. & DBO & $\mathrm{mg} / 1$ & 1,1 & 4,12 & 9,3 \\
4. & Nitratos & $\mathrm{mg} / 1$ & 4,2 & 2,9 & 5,5 \\
5. & Coliformes fecales & $\mathrm{NMP} / 100 \mathrm{ml}$ & 2200 & 400 & 800 \\
6. & Temperatura & ${ }^{\circ} \mathrm{C}$ & 21,9 & 22 & 19,4 \\
7. & Turbidez & $\mathrm{NTU}$ & 25,9 & 17,4 & 47,1 \\
8. & Solidos totales & $\mathrm{gr}$ & 238 & 160 & 316 \\
9. & Fosfatos & $\mathrm{mg} / 1$ & 0,15 & 0,17 & 0,52 \\
\hline
\end{tabular}

Realizado por: Grupo de investigación.

Variables De Alto Impacto Registrados 
Ilustración 2. Variables de mayor impacto del punto 1 de acuerdo con el programa IQAdata.

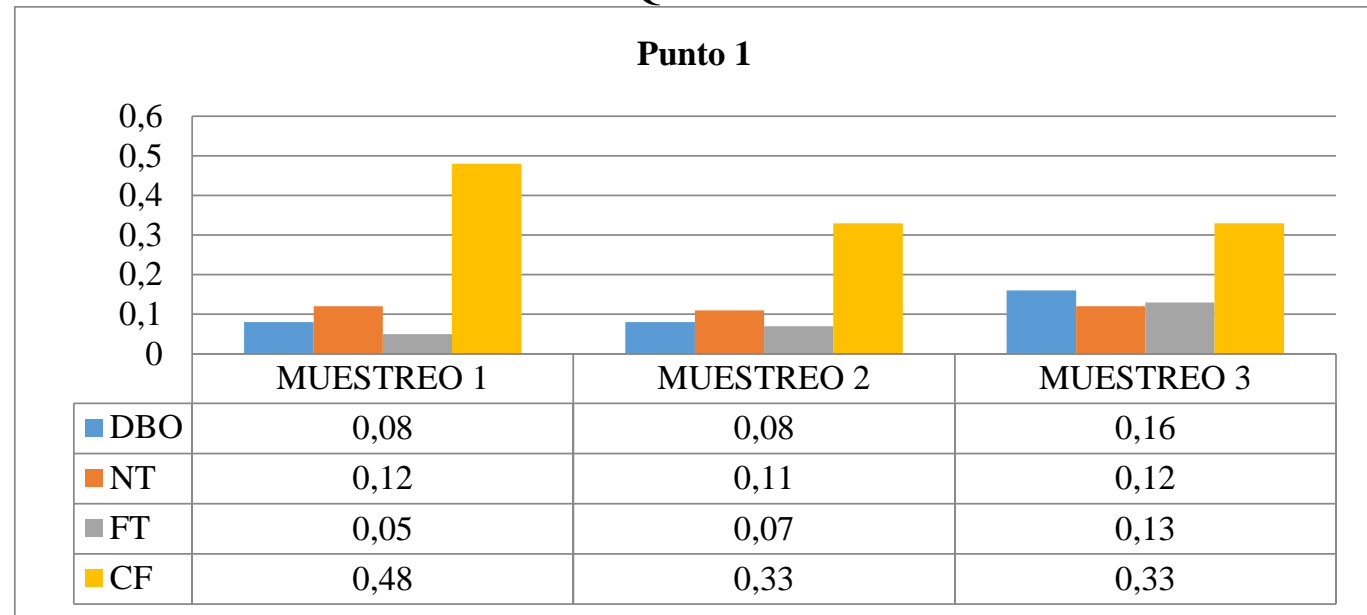

Realizado por: Grupo de investigación.

En la ilustración 3 que corresponde al punto 1 (Barrio la Florida) se puede apreciar claramente que la variable con mayor incidencia son las coliformes fecales representando en el muestreo 1 un valor de 0,48 el mismo que representa un $61 \%$ a la afectación del Índice de calidad de agua, los nitratos con 0,12 son otro de los factores relevantes representando un $15 \%$, la DBO y fosfatos con 0,08 y 0,05 respectivamente representan el $10 \%$ y $6 \%$. El muestreo 2 y 3 nos da un valor de 0,33 que presentan el $51 \%$ y el $40 \%$ de la afectación del ICA respectivamente, en cambio los nitratos nos dan un porcentaje del $17 \%$ y $15 \%$ respectivamente, el DBO en la muestra 2 arroja un resultado de 0,08 y del muestreo 3 de 0,16 dándonos un porcentaje respectivo de $12 \%$ y $20 \%$.

Ilustración 3. Variables de mayor impacto del punto 2 de acuerdo con el programa IQAdata.

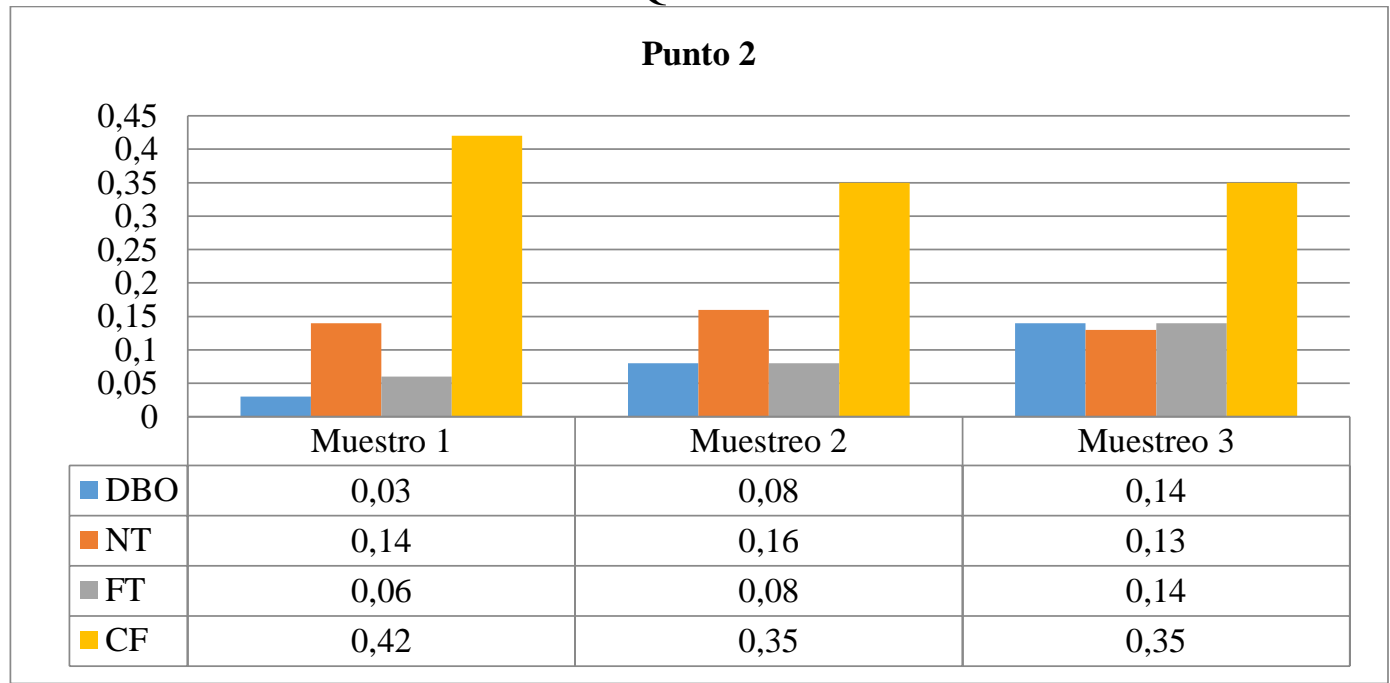

Realizado por: Grupo de investigación. 
En la ilustración 4 que corresponde al punto 2 (Salida al Quílamo) sigue la tendencia en cuanto a los coliformes fecales, siendo la variable de mayor impacto en el muestreo1,2 y 3 de los cuales se obtuvo un valor de 0,$42 ; 0,35$ y 0,35 equivalentes en porcentaje al $58 \%, 46 \%$ y $40 \%$ respectivamente, otra de las variables que más sobresale son los nitratos que varían de 0,$14 ; 0,16$ y 0,13 en los 3 muestreos los mismo que representan un porcentaje de $19 \%$, $21 \%$ y $15 \%$ a la afección del ICA, los fosfatos en los 3 muestreos varían de 0,06; 0,08 y 0,14 siendo en el muestreo 3 el de mayor afección en comparación con el muestreo 1 y 2, los porcentajes respectivos son $8 \%, 11 \%, 16 \%$.

Ilustración 4. Variables de mayor impacto del punto 3 de acuerdo con el programa IQAdata.

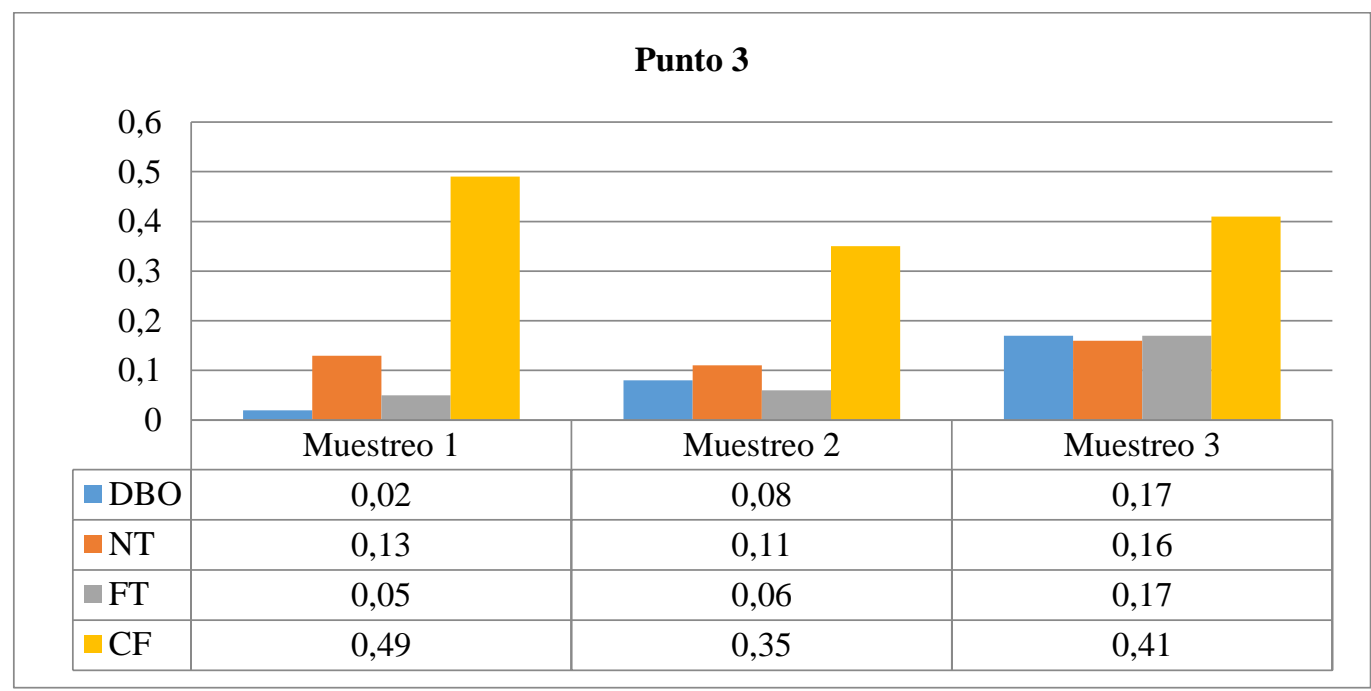

Realizado por: Grupo de investigación.

En la ilustración 5 que corresponde al punto 3 (Puente Salida de Macas) se mantiene la tendencia teniendo en primer lugar a los coliformes fecales como el máximo influyente en la calidad de agua, dándonos valores de 0,$49 ; 0,35$ y 0,41 con porcentajes $60 \%, 52 \%$ y $37 \%$ en las 3 muestras respectivamente, en el caso de los nitratos nos dio valores de 0,$13 ; 0,11$ y 0,16 por lo que se ve representado en los siguientes porcentajes $16 \%, 16 \%$ y $14 \%$, en el muestreo 3 el DBO y fosfatos fueron los de mayor relevancia obteniendo valores de 0,17 en ambos casos, representando un $15 \%$ en la afectación de la calidad del agua.

Como se pudo apreciar en los 3 puntos los coliformes fecales fueron la variable de mayor relevancia para nuestro estudio, resaltando el resultado obtenido de una calidad de agua regular según el ICA_NSF. 


\section{Discusión}

Según (Galárraga, 2000) todos aquellos ríos que están ubicados cerca de zonas urbanas siempre son el punto de contaminación más notorio y por ende entre los parámetros que son más afectados están los coliformes, Nitratos, Fosfatos y DBO, los mismos que son impulsados por la ganadería, agricultura y la misma zona urbana. Los resultados obtenidos nos dicen que la calidad de agua es regular, estos resultados nos hacen suponer que el deterioro en su calidad está íntimamente ligado a dos factores el primero es la actividad antropogénica que no es más que la contaminación por parte de la zona urbana de los barrios; la Florida, 27 de Febrero y el Naranjal por lo que debido a que estos barrios están ubicados a lo largo del tramo de estudio, es decir el río fluye paralelamente a estos barrios los mismo que pueden influir de manera directa o indirecta en la calidad del agua, y el segundo factor puede ser la presencia de ganado, camal del cantón morona que está ubicado en la cercanía del punto 3 de muestreo, durante los meses Marzo a Junio que fue el periodo de estudio se mantuvo un índice de calidad de agua de malo.

Según el Texto unificado de legislación secundaria del (Ministerio del Ambiente, 2015) registro oficial 387 la tabla 1 criterios para las fuentes de aguas para el consumo humano y uso doméstico podemos comparar que los límites permisibles en coliformes fecales son (1000 NPM/100ml) y en nuestro estudio éste parámetro en uno de los muestreos alcanzó los (2000NPM/l) en cuanto DBO5 el límite es $(<2 \mathrm{mg} / \mathrm{l})$ y en el estudio alcanzó un máximo de $(0,17 \mathrm{mg} / \mathrm{l})$, en cuanto a nitratos el límite permisible es de $(50 \mathrm{mg} / \mathrm{l})$, en nuestros resultados se obtuvo $(0,17 \mathrm{mg} / \mathrm{l})$ éstos los parámetros más influyentes dentro nuestro ICA.

\section{Conclusiones.}

- En los puntos 1,2 y 3 durante los tres muestreos realizados según los rangos establecidos por el ICA NFS se determinó que el índice de calidad de agua es REGULAR en el tramo que se estableció el estudio, el parámetro que más influyó en el resultado final del ICA fue coliformes fecales el cual se debe a que se encuentra rodeado de la zona urbana y en ciertos sitios se realizan descargas de aguas servidas al rio y por la actividad ganadera que en sus alrededores se llevan a cabo que por medio de escorrentías son conducidos los contaminantes que tienen como punto final de descarga la microcuenca del rio Jurumbaino, cabe recalcar que los días que se realizaron los muestreos fueron lluviosos.

- En base a los límites permisibles que se encuentra en el TULSMA (Ministerio del Ambiente, 2015) tabla 1 en comparativa con nuestros resultados por el factor coliformes fecales se podría concluir que estas aguas no son aptas para el consumo humano.

\section{Referencias bibliográficas.}

Caho-Rodríguez, C. A., \& López-Barrera, E. A. (2017). Determinación del Índice de Calidad de Agua para el sector occidental del humedal Torca-Guaymaral empleando las 
metodologías UWQI y CWQI. Producción + Limpia, 12(2), 35-49. https://doi.org/10.22507/pml.v12n2a3

Castro, M., Almeida, J., Ferrer, J., \& Diaz, D. (2014). Indicadores de la calidad del agua: evolución y tendencias a nivel global. Ingeniería Solidaria, 10(17), 111-124. https://doi.org/10.16925/in.v9i17.811

Federation, W. E. A. P. H. A. A. W. W. A. (2017). Standard Methods for the Examination of Water and Wastewater. Retrieved from https://www.academia.edu/38769108/Standard_Methods_For_the_Examination_of_ Water_and_Wastewater_23nd_edition

GAD. (2016). Ubicación geográfica | Gobierno Municipal del Canton Morona.

Galárraga, R. (2000). INFORME NACIONAL SOBRE LA GESTION DEL AGUA EN EL ECUADOR Febrero 15 del 2000. Gestion, pp. 1-120. Retrieved from https://www.cepal.org/DRNI/proyectos/samtac/InEc00100.pdf\%0A\%0A

González Meléndez, V., Caicedo Quintero, O., \& Ramirez, N. A. (2013). Aplicación de los índices de calidad de agua NSF , DINIUS y BMWP. Revista Gestión y Ambiente, 16(1), 97-108. Retrieved from http://www.redalyc.org/pdf/1694/169427489003.pdf

Ibarra, G. (2007). El agua o la vida. 9. Retrieved from http://www.elortiba.org/old/pdf/el_agua_o_la_vida.pdf

INEC. (2010). Instituto Nacional de Estadística y Censos - Ecuador.

Instituto Nacional de Meteorología e Hidrología (INAMHI). (2016). Boletin Climatologico Anual 2015. Boletín de Vigilancia Climática Del Ecuador, 1, 31.

Isch, E. (2011). Contaminación de las aguas y políticas para enfrentarla. 52.

Ministerio del Ambiente. (2015). TEXTO UNIFICADO DE LEGISLACIÓN SECUNDARIA DEL MINISTERIO DEL AMBIENTE. Registro Oficial - Edición Especial $\quad \mathrm{N}^{\circ} 387 . \quad$ (097), $21 . \quad$ Retrieved from http://extwprlegs1.fao.org/docs/pdf/ecu155128.pdf

Nelson Fernández, G. R. y F. S. (2009). Una Herramienta Informatica para el Análisis y Valoración de la Calidad del Agua. D- Universidad de Pamplona, 2, 11. Retrieved from https://ebookcentral.proquest.com/lib/espochsp/detail.action?docID=3177667

Noticias Multinivel. (2019). Que es NSF International - Información sobre NSF International. Retrieved June 7, 2019, from https://noticiasmultinivel.com/nsfinternational/ 
NSF Internacional. (2004). Who Is NSF International - NSF International.

Posselt, E., \& Costa, A. (2015). AB software IQAData 2015. registro con el INPI N¹06702, Programa de Maestría en Sistemas y Procesos Industriales PPGSPI, UNISC,2015. 2015. Retrieved from http://www.unisc.br/ppgspi

Ramírez, C. A. S. (2011). Calidad del agua: evaluación y diagnóstico (1 edicion; L. D. L. Escobar, Ed.). Retrieved from https://ebookcentral.proquest.com/lib/espochsp/reader.action?docID=3198982

Santiago, L., \& Fernández, Q. (2017). Aplicacion del Indice de calidad de agua en el rio Portoviejo, Ecuador. XXXVIII(3), 41-51.

Torres, P., Hernán, C., Paola, C. •, \& Patiño, J. (2009). WATER QUALITY INDEX IN SURFACE SOURCES USED IN WATER PRODUCTION FOR HUMAN CONSUMPTION. A CRITICAL REVIEW. Ingenierias Universidad de Medellin, 8(1692-3324), 79-94. 
PARA CITAR EL ARTÍCULO INDEXADO.

Ureta Valdez, R., Méndez Zambrano, P., \& Cazar Rivera, E. (2019). Influencia de la zona urbana de Macas en el Índice Calidad de Agua del río Jurumbaino. Ciencia Digital, 3(3.1), 102-114. https://doi.org/10.33262/cienciadigital.v3i3.1.679

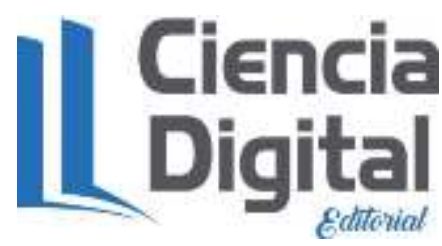

El artículo que se publica es de exclusiva responsabilidad de los autores y no necesariamente reflejan el pensamiento de la Revista Ciencia Digital.

El artículo queda en propiedad de la revista y, por tanto, su publicación parcial y/o total en otro medio tiene que ser autorizado por el director de la Revista Ciencia Digital.
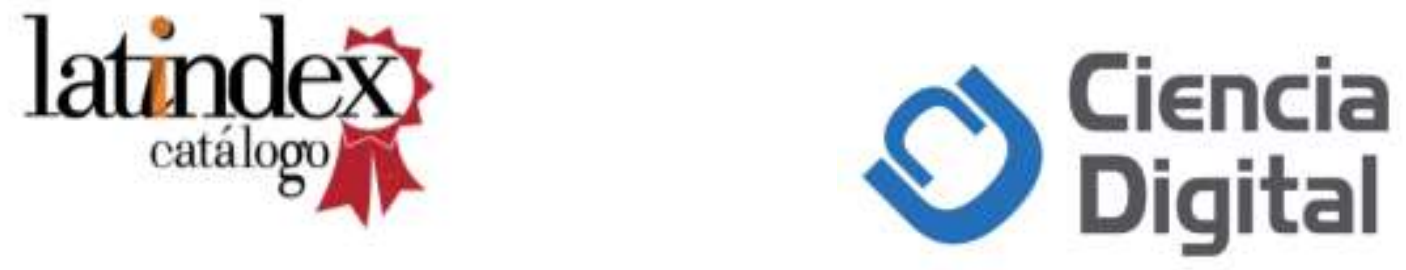